\title{
To Study Efficacy of Blood Pressure Management Program (BPMP) in Male Elderly Patients with Known Case of Hypertension: An Observational Study
}

\author{
Rohit Sane $^{1}$, Sagar Patil ${ }^{2}$, Nita Hinge ${ }^{3}$, Nilesh Kulthe ${ }^{4}$, Swati Ambulkar ${ }^{5}$, Rahul Mandole ${ }^{1, ~ * ~}$ \\ ${ }^{1}$ Department of Research and Development, Madhavbaug Cardiac Care Clinics and Hospitals, Mumbai, India \\ ${ }^{2}$ Madhavbaug Cardiac Care Clinics, Mumbai, India \\ ${ }^{3}$ Madhavbaug Cardiac Care Clinics, Pune, India \\ ${ }^{4}$ Department of Medical Operations, Madhavbaug Cardiac Care Clinics and Hospitals, Mumbai, India \\ ${ }^{5}$ Department of Medical Operations, Madhavbaug Cardiac Care Clinics and Hospitals, Pune, India
}

Email address:

cromilagro@gmail.com (R. Mandole)

*Corresponding author

\section{To cite this article:}

Rohit Sane, Sagar Patil, Nita Hinge, Nilesh Kulthe, Swati Ambulkar, Rahul Mandole. To Study Efficacy of Blood Pressure Management Program (BPMP) in Male Elderly Patients with Known Case of Hypertension: An Observational Study. Cardiology and Cardiovascular Research. Vol. 2, No. 3, 2018, pp. 49-54. doi: 10.11648/j.ccr.20180203.11

Received: July 19, 2018; Accepted: July 30, 2018; Published: October 12, 2018

\begin{abstract}
Around $20 \%$ of the population are in prehypertension stage, and nearly $40 \%$ of the adult population from Hypertension (HTN). Blood Pressure Management Program (BPMP) is an Ayurvedic treatment strategy using Panchakarma. This study was conducted to evaluate the effect of BPMP on systolic blood pressure (SBP), diastolic blood pressure (DBP), mean arterial pressure (MAP), body mass index (BMI) and dependency on conventional therapy in elderly male patients of HTN. This observational study was conducted from January 2017 to February 2018, wherein the data of HTN patients who attended Madhavbaug clinics in Maharashtra, India were identified. Data of patients who were administered BPMP (60-75 minutes) with minimum 6 sittings over 90 days ( \pm 15 days) were considered. Variables were compared between day 1 and day 90 of BPMP. Out of 29 enrolled patients, 24 were finally selected for analysis. BPMP showed significant improvement in SBP by 24.66 (from $150.67 \pm 12.97$ to $126 \pm 13.01 ; \mathrm{p}<0.001$ ), DBP by 10.8 (from $87.7917 \pm 7.72$ to $76.917 \pm 7.59, \mathrm{p}<0.001$ ), MAP by 15.4 (from $108.75 \pm 7.14$ to $93.25 \pm 8.72$, $\mathrm{p}<0.001)$. BMI $(25.7275 \pm 2.63 \mathrm{~kg} / \mathrm{m} 2$ to $24.91 \pm 2.32 \mathrm{~kg} / \mathrm{m} 2$ ), also showed significant reduction. Dependency on concomitant medicines was reduced, with the number of patients on no concomitant medicines increasing from $19 \%$ to $29 \%$. BPMP can serve as an effective therapeutic regiment to combat HTN in elderly male patients.
\end{abstract}

Keywords: Blood Pressure Management Program, BPMP, Panchakarma, Hypertension, Systolic, Diastolic, Mean Arterial Pressure, Elderly Male

\section{Introduction}

Hypertension (HTN) has assumed epidemic level prevalence rates in past few decades. Around $20 \%$ of the population are in prehypertension stage, and nearly $40 \%$ of the adult population from HTN [1]. Same scenario is reflected in mortality rates due to HTN, which was estimated to 10 million deaths in 2010. It is estimated that HTN is the main culprit of stroke, heart failure and other heart diseases in half of the patients [2]. Due to increased awareness in developed nations, prevalence of HTN has not increased in past few years, but it has shown consistent rise in developing countries like India [3]. Not only less accessible healthcare delivery system, but also less diagnosis rates also add to increased burden of the disease in developing countries. Uncontrolled/poorly controlled HTN frequently leads to fatal and nonfatal complications like haemorrhagic stroke, myocardial infarction, renal failure, etc [4]. Apart from these, 
HTN contributes to a major chunk of financial burden of health expenses on a national scale, since it leads to loss of earnings, disability, premature mortality, etc [5].

Conventionally, HTN is diagnosed by systolic blood pressure $>140 \mathrm{~mm} \mathrm{Hg}$ (SBP) and diastolic BP (DBP) > $100 \mathrm{~mm} \mathrm{Hg}$ [6]. Variety of drugs are available for treatment of HTN, which include beta blockers, angiotensin converting enzyme inhibitors (ACEIs), diuretics, calcium channel blockers, etc [7]. But these drugs frequently give rise to number of adverse effects like hypotension, worsening of asthma, heart block, dizziness, headache, hallucinations, etc [8]. Moreover, the management of HTN is complex due to vitality of consideration of numerous factors like comorbidities, age. Despite the availability of all the working guidelines for management of HTN and extensive lists of conventional medicines, HTN is continuously creating havoc. Therefore, we need some form of effective therapy which can ameliorate ill effects of HTN and also overcome shortcomings of conventional therapy, which can increase the quality of life in hypertensive patients [9].

Similar to the action of conventional medicines, many herbal drugs have been found to be effective in reducing BP in HTN $[10,11]$. BP Management Program (BPMP) is an Ayurvedic antihypertensive management program which combines Panchakarma with Diet therapy in patients of HTN. Following detoxifying procedures are used in BPMP $[12,13]$ :

I. Snehana/External Massage Therapy,

II. Swedana/ Passive Heat Therapy,

III.Shirodhara/Dripping Oil on Forehead.

Given the BP reducing action of herbal drugs and beneficial effects of Panchakarma in HTN, we planned this observational study to analyze the effect of BPMP on SBP, DBP, mean arterial pressure (MAP), body mass index (BMI), dependency of elderly male hypertensive patients on conventional antihypertensive medicines at the end of the study period.

\section{Subjects and Methods}

\subsection{Study Design}

Retrospective record based Observational study

\subsection{Sample Size}

Total Number of Subjects: 24

\subsection{A Number of Screened Patients}

Total 29 hypertensive elderly patients were enrolled in HTN program. Out of which we have observed the efficacy of BPMP in 24 patients' data which were elderly (age $=\geq 60$ years) male patients.

\subsection{Duration of Study}

January 2017 to February 2018

\subsection{Study Site}

Madhavbaug Clinics from all over Maharashtra

\subsection{Inclusion Criteria}

We have observed the efficacy of HTN program in:

i. Elderly male patients $=$ Age $\geq 60$ years

ii. Patient with past history of HTN.

\subsection{Methodology}

Methodology adopted for the present study is depicted in figure 1 .

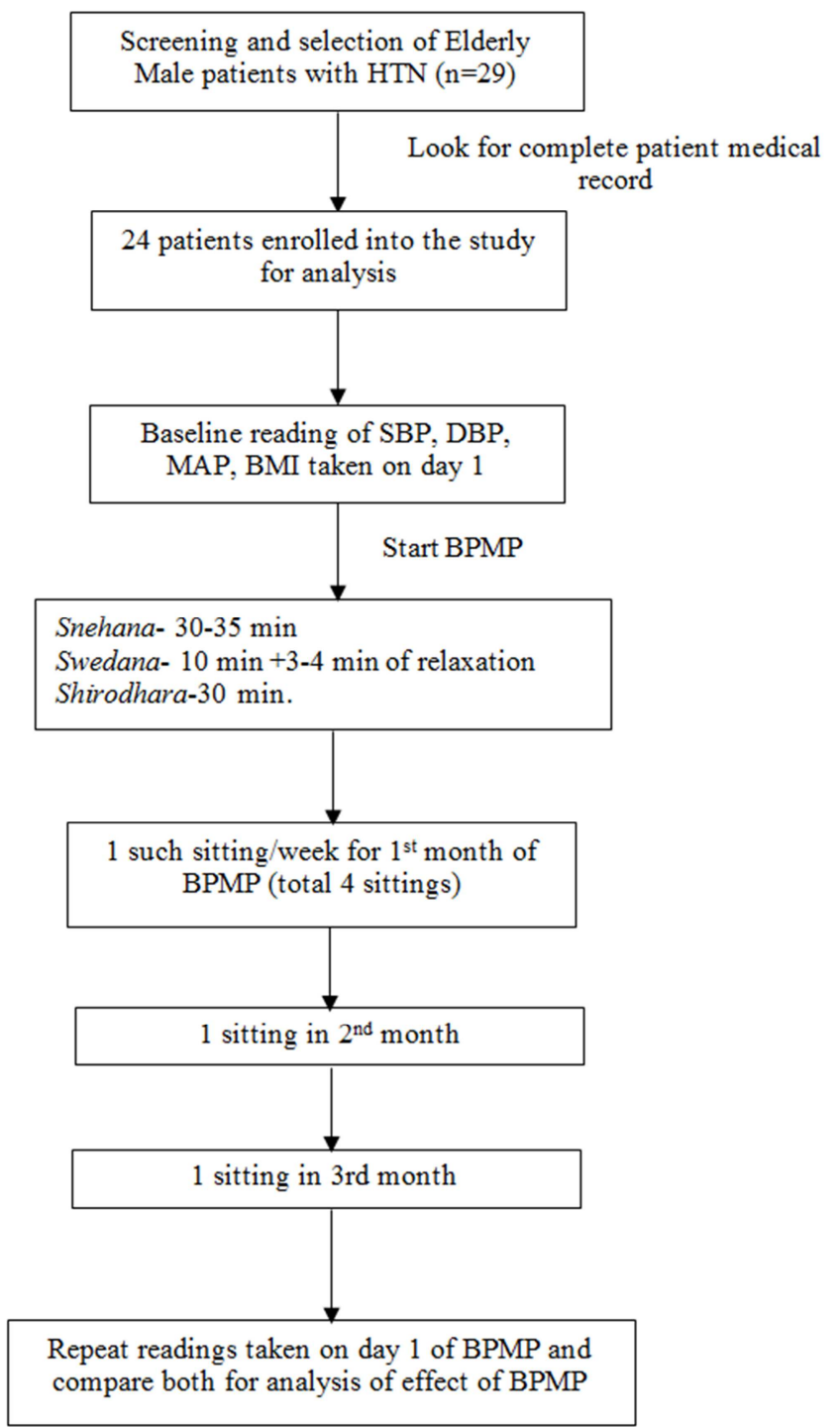

Figure 1. Methodology adopted for the present study.

The BPMP is a 3-step procedure which was performed on the patients of HTN after a light breakfast. One sitting of the procedure took 65-75 minutes, as described in table 1 [12, $13]$. 
Table 1. Study Treatment: Blood pressure management program (HTN Kit).

\begin{tabular}{|c|c|c|c|}
\hline Step of BPMP & Type of Therapy & Herbs used for therapy & Time Duration of Therapy \\
\hline Snehana & $\begin{array}{l}\text { Massage or external oleation (centripetal } \\
\text { upper strokes directed towards heart) }\end{array}$ & It is $2 \%$ Rose flavoured Sesame oil. & 30-35 minutes \\
\hline Swedana & Passive heat therapy & $\begin{array}{l}\text { Dashmoola (group of ten herbal roots) with steam } \\
\text { at } \leq 40 \text { degrees Celsius) }\end{array}$ & $\begin{array}{l}10-15 \text { minutes }+3-4 \text { minutes of } \\
\text { relaxation after procedure }\end{array}$ \\
\hline Shirodhara & $\begin{array}{l}\text { Decoction dripping therapy from a height } \\
\text { of } 7-8 \mathrm{~cm}\end{array}$ & $\begin{array}{l}100 \mathrm{ml} \text { of Luke-warm Nardostachys jatamansi } \\
\text { decoction }\end{array}$ & 30 minutes \\
\hline
\end{tabular}

On day 1 of BPMP, the patients had undergone BP measurement as per JNC VIII guidelines [14]. Three readings were taken few minutes apart, and average of the 3 was considered for analysis (baseline reading). This process was repeated on day 90 of BPMP to calculate the change from baseline reading. The BMI for day 1 and day 90 of the patients was calculated by checking the weight and the height from the medical data sheets of patients and using the formula: weight in kilograms/ (height in meters) ${ }^{2}$. The dependency on standard medication was calculated both on day 1 and day 90 of BPMP as the percentage of patients out of the total enrolled ones who required a conventional allopathic therapeutic agent during the study period of 90 days.

\subsection{Statistical Analysis}

Data were pooled and coded in Microsoft Excel spreadsheet Paired $\mathrm{T}$ test were used to test statistical significance for Primary End Points - Reduction in Blood pressure (SBP and DBP) and Secondary End Point Reduction in BMI and MAP after 90-day follow-up as compared to day 1 (baseline). We used R (Version 3.5.0) software and excel to analyse the data.

\section{Results}

Study population:

A total of 29 patients' data was screened for inclusion in the study. However, based on the availability of data (Day 1 and Day 90) and the inclusion criteria, 24 patients were selected, and their data was considered for analysis. The baseline characteristics of these patients are shown in table 2 .

Table 2. Baseline characteristics of the study subjects $(n=24)$.

\begin{tabular}{lll}
\hline Patients & $\mathbf{2 4}$ & \\
\hline Age (Years) & $\mathbf{6 6 . 5} \pm \mathbf{7 . 8 6}$ & \\
\hline & Day 1 & Day 90 \\
\hline SBP & $150.67 \pm 12.97$ & $126 \pm 13.01$ \\
DBP & $87.7917 \pm 7.72$ & $76.917 \pm 7.59$ \\
BMI & $25.7275 \pm 2.63$ & $24.91 \pm 2.32$ \\
MAP & $108.75 \pm 7.14$ & $93.25 \pm 8.72$ \\
\hline
\end{tabular}

SBP, Systolic blood pressure; DBP, Diastolic BP; BMI, Body Mass Index; MAP, Mean Arterial Pressure.

Note: Categorical data were expressed in terms of percentage and continuous data were expressed as Mean \pm SD

Consumption of allopathic medicines on days 1 and 90 were as shown in Table 3. The participants dependent on allopathic medicines were reduced after 90 days. The participants on medications such as angiotensin II receptor blockers (ARBs), beta-blockers, calcium channel blockers (CCBs) were notably condensed after 90 days. The graphical representation of consumption of allopathic medicines on days 1 and 90 is depicted in Figure 2.

Comparison of clinical parameters between baseline values and $90^{\text {th }}$ day were shown in Table 4. Mean Arterial Pressure (MAP) $(P<0.001)$, Body Mass Index $(\mathrm{P}<0.001)$, Systolic Blood Pressure (SBP) $(P<0.001)$, Diastolic Blood Pressure $(P<0.001)$ were reduced and significantly improved after the treatment, i.e., after 90 days. The graphical representation of the same is represented in Figure 3.

Table 3. Consumption of allopathic medicines on days 1 and 90.

\begin{tabular}{|c|c|c|c|c|}
\hline & Baseline & Percentage & After 90 days & Percentage $(\%)$ \\
\hline NSAID & 2 & 9.52 & 1 & 4.76 \\
\hline $\mathrm{ARB}$ & 11 & 52.38 & 7 & 33.33 \\
\hline Antiplatelet & 1 & 4.76 & 0 & 0.00 \\
\hline Beta blocker & 8 & 38.10 & 3 & 14.29 \\
\hline $\mathrm{CCB}$ & 6 & 28.57 & 5 & 23.81 \\
\hline Statin & 2 & 9.52 & 0 & 0.00 \\
\hline $\mathrm{ACE}$ & 2 & 9.52 & 1 & 4.76 \\
\hline Thiazide Diuretic & 6 & 28.57 & 4 & 19.05 \\
\hline Biguanide & 3 & 14.29 & 3 & 14.29 \\
\hline Sulfonylurea & 2 & 9.52 & 2 & 9.52 \\
\hline No Medicine & 4 & 19.05 & 6 & 28.57 \\
\hline NSAID + antiplatelet & 1 & 4.76 & 0 & 0.00 \\
\hline
\end{tabular}




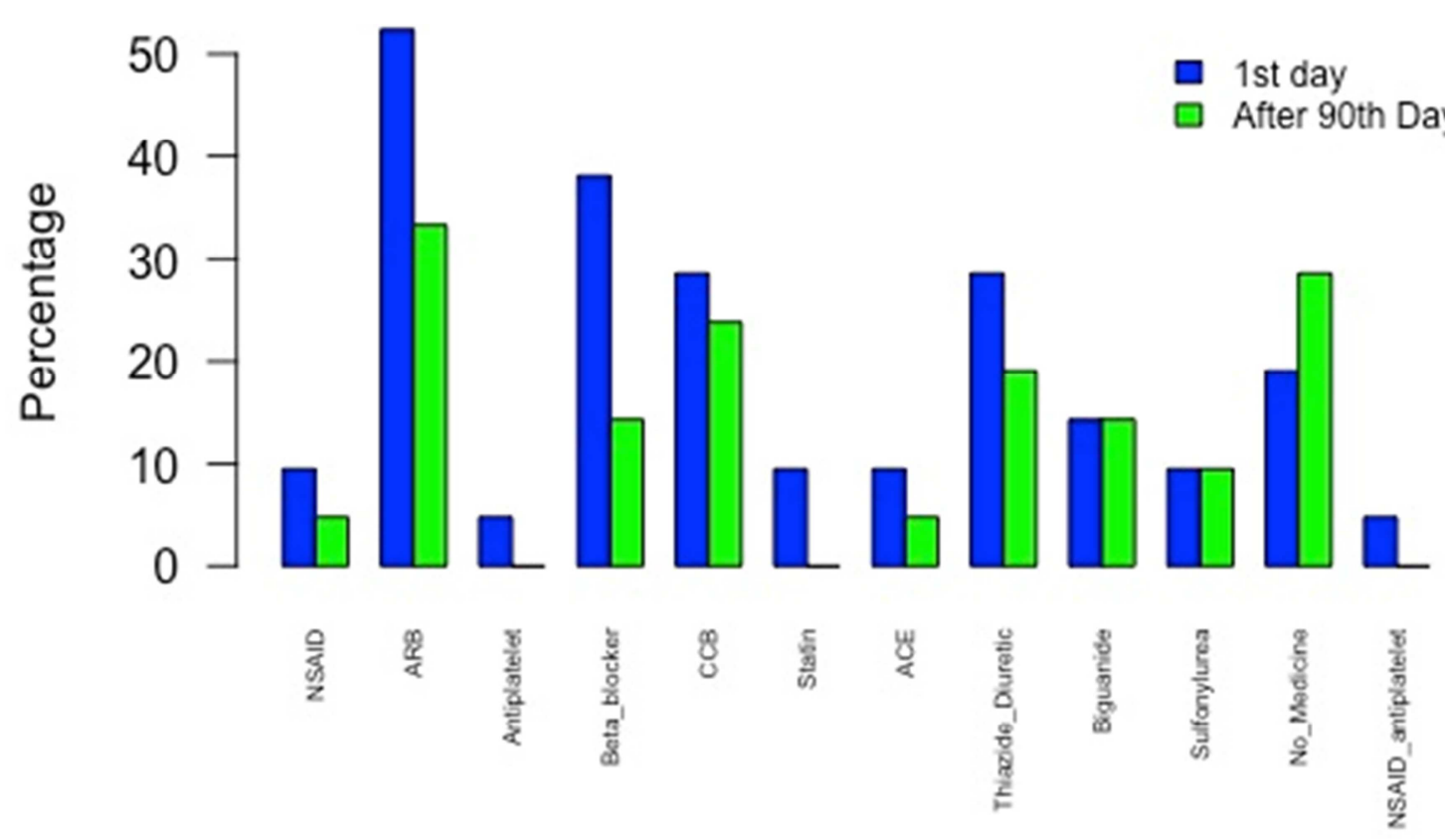

Figure 2. Consumption of allopathy medicines at days 1 and 90 days $(n=24)$.

Table 4. Comparison of clinical parameters between baseline values and $90^{\text {th }}$ day.

\begin{tabular}{llll}
\hline Variable (n=24) & Baseline (day 1) & After 90 days & Difference \\
\hline SBP & $150.67 \pm 12.97$ & $126 \pm 13.01$ & 24.66667 \\
DBP & $87.7917 \pm 7.72$ & $76.917 \pm 7.59$ & 10.875 \\
BMI & $25.7275 \pm 2.63$ & $24.91 \pm 2.32$ & 0.821667 \\
MAP & $108.75 \pm 7.14$ & $93.25 \pm 8.72$ & $<0.001 * * *$ \\
\hline
\end{tabular}

***Highly significant; MAP, Body Mass Index; SBP, DBP

Fig 3.1: Comparison of SBP

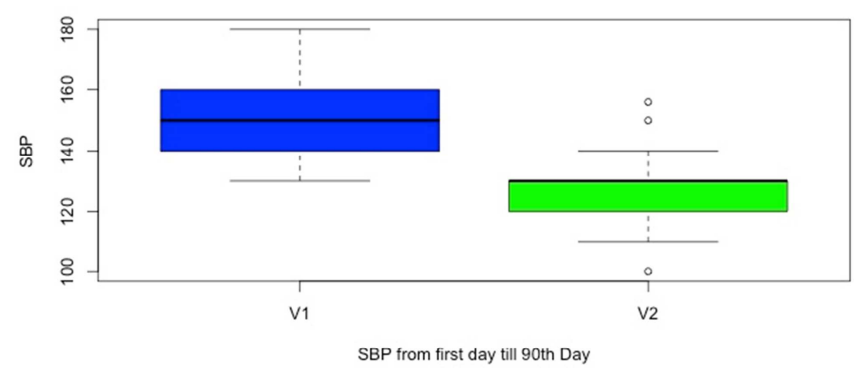

Fig 3.3: Comparison of BMI

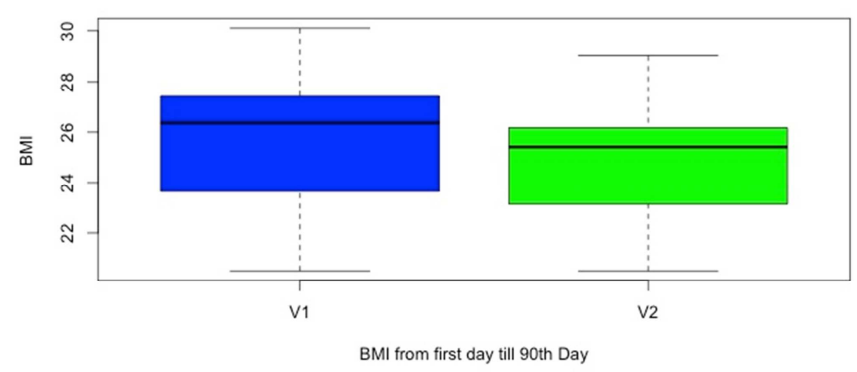

Fig 3.2: Comparison of DBP

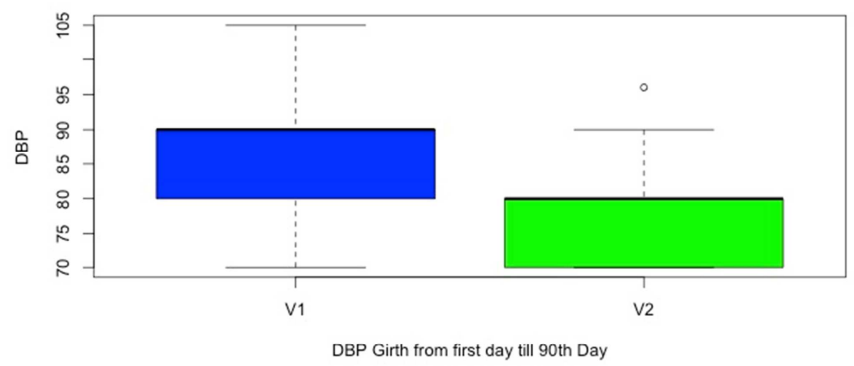

Fig 3.4: Comparison of MAP

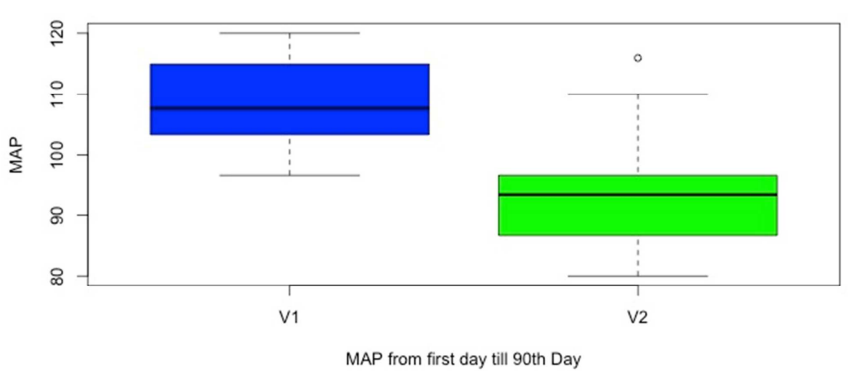

Figure 3. Comparison of clinical parameters between baseline values and $90^{\text {th }}$ day.

\section{Discussion}

HTN is the leading cause of cardiovascular deaths all over the world. Troublesome fact is that its prevalence is still increasing at alarming rates, especially in India. This is happening, despite the accessibility of numerous conventional drugs like beta blockers, diuretics, etc. and 
guidelines for the treatment of HTN. Conventional medicines act like a double edged sword; their extensive adverse effect profile and increased cost of consumption adds tremendously to burden of disease in society. That is why we need such a therapy which can ameliorate all these drawbacks of conventional drugs and maintaining the same efficacy of conventional drugs.

Conventional antihypertensive drugs act in HTN by reducing BP via:

i. Reduction in sympathetic tone,

ii. Anti-inflammatory action,

iii. Vasodilation,

iv. Antioxidant,

v. Reduction in sodium and water load [7].

Many herbal drugs act through above mechanism and also they have added advantage of less/ no adverse effects and less cost of therapy, as compared to conventional medications. Therefore, Panchakarma along with herbal drugs might serve as an effective alternate option for management of HTN. Panchakarma techniques used in BPMP itself have BP lowering effects by calming effect, thus reducing sympathetic overactivity, reduction in sodium and water load, etc $[12,13]$. Also herbal drugs used in Panchakarma under BPMP have following beneficial effects in reducing BP by:

i. Rose fragrance can decrease sympathetic activity. The probable mechanism responsible for this beneficial effect might be relaxation through inhalation of pleasant smell, and increased binding of essential oil component to GABA (Gamma-Aminobutyric acid) inhibitory receptors in brain, which reduce sympathetic stimulation [15].

ii. Nardostachys jatamasi- Reduction of stress, antioxidant, cardio protective effects [16].

Also, it has been found that increased BMI is epidemiologically linked with increased occurrence of a variety of diseases like obesity, HTN, heart failure, atrial fibrillation, etc. BPMP resulted in significant reduction (high statistical significance) in BMI along with SBP, DBP, MAP. Thus, it combats all the parameters related to HTN.

Instead of an isolated measurement of SBP and DBP, some researchers favor the measurement of MAP for assessing the efficacy of antihypertensive drugs, since it takes into account both SBP as well as DBP. MAP act as better prognosticator in presence of multiple cardiovascular risk covariates [17]. In our study there was significant reduction in MAP, which suggest that BPMP might serve as an effective tool to combat HTN. Another advantage of BPMP, which we found in our study was that, it reduced dependency of patients on conventional medications. This is a more important finding for population like India, where adverse effects and increased cost of therapy greatly reduces compliance and adherence to therapy, thus increasing complications of HTN [9].

Despite all these significant positive findings of BPMP, we recommend similar studies with a larger number of study participants and increased duration of follow up, so that the findings of our study can be corroborated and forecasted to larger populations.

\section{Conclusion}

Since HTN is multifactorial disease, it is important to combat the derangement in all the related parameters, namely SBP, DBP, MAP, BMI. In the light of effects of BPMP in our present study on these parameters, it is fair anticipation that BPMP will serve as an effective therapeutic option for treatment of HTN in elderly male patients.

\section{Acknowledgements}

The authors thank the study participants and their families, without whom this study would not have been accomplished.

\section{References}

[1] Suma R, Mayamol T, Divakaran B, et al. Hypertension: prevalence, awareness, treatment and control in a rural area of North Kerala, India. Int J Community Med Public Health. 2017;4:3561-7.

[2] Campbell N, Lackland D, Niebylski M. High Blood Pressure: Why Prevention and Control Are Urgent and Important-A 2014 Fact Sheet From the World Hypertension League and the International Society of Hypertension. J Clin Hypertens. 2014; 16(8):551-3.

[3] Kearney P, Whelton M, Reynolds K, et al. Worldwide prevalence of hypertension: a systematic review. J Hypertens. 2004; 22(1):11-9.

[4] Noblet A, Lopes M, Lopes G, et al. Complications of Hypertension in Men and Women Seen in a Referral Outpatient Care Unit. Arquivos Brasileiros de Cardiologia. 2004; 83(4): 314-9.

[5] Day W. World Health Day 2013. A Glob Br Hypertens. 2013. Accessed on 4 July 2017.

[6] National Heart Foundation of Australia. Guidelines for diagnosis and management of hypertension in adults-2016. Melbourne: National Heart Foundation of Australia, 2016.

[7] Drug Treatment for People with Hypertension. Retrieved from https://www.pco.gov.hk/english/resource/files/professionals_H T_Module7.pdf

[8] Doshi V, Dahake A, Suthar A. Adverse Effects Associated with the Use of Antihypertensive Drugs: An Overview. International journal of Pharmtech Research. 2010; 2(1):10-13.

[9] Pangtey R, Meena G. Impact of hypertension on quality of life among people living in an urban area of Delhi, India. Journal of hypertension. 2016; 34(e1): e462.

[10] Tabbasum N, Ahmad F. Role of natural herbs in the treatment of hypertension. Pharmacognosy Reviews. 2011; 5(9): 30-40.

[11] Agrawal M, Nandini D, Sharma V, et al. Herbal remedies for treatment of hypertension. International Journal of Pharmaceutical Sciences and Research. 2010; 1(5):1-21.

[12] Choudhary K, Sharma P, Sharma V. Hypertension and its management through panchakarma. Journal of Ayurveda and Holistic Medicine. 2015; 3(3):28-31. 
[13] Vithalani L, Dalvi S, Lele V, et al. Hypertension - an ayurvedic perspective. International Journal of Ayurvedic Medicine. 2015; 3(11):2322-2329.

[14] Olin B. Hypertension: The Silent Killer: Updated JNC-8 Guideline Recommendations Associate Clinical Professor of Pharmacy Practice, Drug Information and Learning Resource Center. 2015.

[15] Haze S, Sakai K, Gozu Y. Effects of fragrance inhalation on sympathetic activity in normal adults. Jpn J Pharmacol. 2002; 90: 247-253.
[16] Sahu R, Dhongade H, Pandey A, et al. Medicinal Properties of Nardostachys jatamansi (A Review). Oriental Journal of Chemistry. 2016;32(2): 859-866.

[17] Kundu R, Biswas S, Das M. Mean Arterial Pressure Classification: A Better Tool for Statistical Interpretation of Blood Pressure Related Risk Covariates. Cardiology and Angiology: An International Journal. 2017; 6(1):1-7. 